\title{
Rapid and Simple Method for the Determination of Pregabalin in Human Plasma using Liquid Chromatography-tandem Mass Spectrometry (LC-MS/MS): Application to a Bioequivalence Study of Daewoong Pregabalin Capsule To Lyrica ${ }^{\circledR}$ Capsule (Pregabalin $150 \mathrm{mg}$ )
}

\author{
Ki-Ho Jang ${ }^{1,2}$, Ji-Hyung Seo ${ }^{1,2}$, Sung-Vin Yim $^{2}$ and Kyung-Tae Lee ${ }^{1,2 \uparrow}$ \\ ${ }^{1}$ College of Pharmacy, Kyung Hee University, Hoegi-Dong, Dongdaemun-Gu, Seoul, 130-701, Korea \\ ${ }^{2}$ College of Medicine, Kyung Hee University, Hoegi-Dong, Dongdaemun-Gu, Seoul, 130-701, Korea
}

(Received July 7, $2011 \cdot$ Accepted August 10, 2011)

\begin{abstract}
Method using liquid chromatography coupled with tandem mass spectrometry (LC-MS/MS) was developed and validated for the determination of pregabalin in plasma samples. Acquisition was performed by monitoring the transitions: $m / z \quad 160.1 \rightarrow 142.2$ for pregabalin and $m / z 423.2 \rightarrow 207.1$ for losartan (as an internal standard). After cold acetonitrileinduced protein precipitation of the plasma samples, separation was performed with $\mathrm{C}_{18}$ column by isocratic mobile phase consisted of $10 \mathrm{mM}$ ammonium acetate and acetonitrile (15:85, v/v). Results were linear over the concentration ranged from 0.1 to $10 \mu \mathrm{g} / \mathrm{mL}$ and the correlation coefficients $(r)$ were $\geq 0.99$. Intra- and inter-day precisions were $\leq 6.02$ and $\leq 11.04 \%$, respectively, and intra- and inter-day accuracies were 96.60-101.09 and 98.10-102.60\%, respectively. This validated method was successfully applied to a bioequivalence study of two formulations of pregabalin, Daewoong pregabalin capsule (Daewoong Pharm. Co., Ltd.) and Lyrica ${ }^{\circledR}$ capsule (Pfizer Korea Ltd.) in twenty eight healthy Korean volunteers. The subjects received a single oral dose of each formulation $(150 \mathrm{mg}$ as pregabalin) in a randomized $2 \times 2$ crossover study and plasma samples were obtained from each subject at predetermined time intervals. Then, the pharmacokinetic parameters $\left(\mathrm{AUC}_{0-\mathrm{t}}\right.$, $\mathrm{C}_{\max }$ and $\mathrm{T}_{\max }$ ) were calculated and statistically analyzed to assess the differences between two formulations. The $90 \%$ confidence intervals for the log-transformed data were acceptable range of $\log 0.8$ - $\log 1.25$ (e.g., $\log 1.0048$ - $\log 1.0692$ for $\mathrm{AUC}_{0-\mathrm{t}}, \log 0.9142-\log 1.0421$ for $\mathrm{C}_{\max }$ ). Thus, $\mathrm{AUC}_{0-\mathrm{t}}$ and $\mathrm{C}_{\max }$ met the criteria of the Korea Food and Drug Administration (KFDA) for bioequivalence test indicating that Daewoong pregabalin capsule was bioequivalent to Lyrica ${ }^{\circledR}$ capsule.
\end{abstract}

Key words - Pregabalin, Daewoong pregabalin capsule, Lyrica ${ }^{\circledR}$ capsule, Bioequivalence study, Liquid chromatographytandem mass spectrometry

Pregabalin, (S)-3-aminomethyl-5-methyl hexanoic acid, a member of $\gamma$-aminobutyric acid (GABA) analogues, crosses the blood brain barrier and potentially binds to the $\alpha_{2}-\delta$ subunit of the voltage-sensitive calcium channel in CNS (Zareba, 2008). Potent binding at this site reduces calcium influx at hyperexcited nerve terminals, and reduces the release of several neurotransmitters, including glutamate, noradrenaline, and substance P (Dooley et al., 2002; Maneuf et al., 2001; Gee et al., 1996). Based on these activities, pregabalin was approved for the treatment of: neuropathic pain, partial seizure and generalized anxiety disorder (Zareba, 2008; Gray, 2007; Framton and Foster, 2005).

There are few published papers for pharmacokinetics of pregabalin. After oral administration, pregabalin is rapidly absorbed through the intestinal tract with peak human plasma concentrations $\left(\mathrm{C}_{\max }\right)$ occurring at $1 \mathrm{hr}$ and its bioavailability is

\footnotetext{
Corresponding Author :

Tel : +82-2-961-0860, E-mail : ktlee@khu.ac.kr

DOI : $10.4333 /$ KPS.2011.41.4.255
}

approximately $\geq 90 \%$. Pregabalin does not bind to plasma protein and its apparent volume of distribution $\left(\mathrm{V}_{\mathrm{d}}\right)$ after oral administration is approximately $0.5 \mathrm{~L} / \mathrm{kg}$. Less than $2 \%$ of the dose is metabolized and the rest of dose is excreted unchanged in urine. The mean terminal disposition half life $\left(t_{1 / 2}\right)$ ranged from 4.8 to $6.3 \mathrm{hr}$ in healthy human volunteers with normal renal function and renal clearance ranged from 67 to $81 \mathrm{~mL} /$ $\min$ (Guay, 2005). The $\mathrm{C}_{\max }$ has been reported to be 4.04$4.11 \mu \mathrm{g} / \mathrm{mL}$ and the time values achieved $\left(\mathrm{T}_{\max }\right)$ were 1.17$1.25 \mathrm{hr}$ after the oral administration of the $150 \mathrm{mg}$ of pregabalin capsule in twelve healthy human volunteers (Vaidya et al., 2007). In other study, after the oral administration of a single dose $300 \mathrm{mg}$ capsule of pragabalin in eighteen healthy human volunteers, the mean value of area under the plasma concentration-time curve $\left(\mathrm{AUC}_{0-\mathrm{t}}\right)$ has been reported to be 43.08-48.77 $\mu \mathrm{g} \cdot \mathrm{hr} / \mathrm{mL}$. The $\mathrm{C}_{\max }$ ranged from 6.93-7.48 $\mu \mathrm{g} /$ $\mathrm{mL}$ at $1.86-2.12 \mathrm{hr}\left(\mathrm{T}_{\max }\right)$ (Mandal et al., 2008). However, there had been no reported studies of pharmacokinetic analysis of pregabalin in Korean.

Several analytical methods have been reported for the deter- 
mination of pregabalin in pharmaceutical preparations and biological fluids by LC-UV (Kasawar and Farooqui, 2010; Gujral et al., 2009) or LC-fluorescence (Dousa et al., 2010; Vermeij and Edelbroek, 2004). These methods require pre-column derivatization of analyte with $o$-phthaldialdehyde (OPA) and 3mercaptopropionic acid or picrylsulfonic acid. In application, derivatization may be difficult and gives inaccurate estimation of analyte due to incomplete derivatization if reaction conditions are not strictly maintained. Therefore, we developed a rapid and simple method for the determination of pregabalin in human plasma using liquid chromatography coupled with tandem mass spectrometry (LC-MS/MS). This method was fully validated and successfully used to assess the bioequivalence of two formulations of pregabalin after the oral administration of a single dose $150 \mathrm{mg}$ capsule in twenty eight healthy Korean volunteers, where these pharmacokinetic parameters were not yet reported in Korean population.

\section{Materials and Methods}

\section{Materials and instruments}

The test formulation (Daewoong pregabalin capsule, $150 \mathrm{mg}$ as pregabalin, Daewoong Pharm. Co., Ltd., Korea, lot no. 960524; expiration date, November 7th 2013), which was manufactured in accordance with the Korean Good Clinical Practice (KGCP) guidelines (KFDA, 2009), and the reference formulation (Lyrica ${ }^{\circledR}$ capsule, $150 \mathrm{mg}$ as pregabalin, Pfizer Korea Ltd., Korea, lot no. 0516119; expiration date, November 5th 2012) were supplied in the form of capsules.

Pregabalin (99.2\% purity) was supplied from Daewoong Pharm. Co., Ltd., (Gyunggi-Do, Korea) and losartan potassium was supplied from Daewoo Pharm. Co., Ltd., (Busan, Korea), respectively. Chemical structures of pregabalin and losartan (internal standard, IS) are presented in Figure 1. HPLC-grade acetonitrile and methanol were purchased from J.T. Baker (Phillipsburg, NJ, USA). Ammonium acetate was purchased from Sigma-Aldrich (St. Louis, MO, USA) and o-phosphoric acid was purchased from Wako (Osaka, Japan). Water was obtained from a Milli-Q water purification system (Millipore Corp., Bedford, MA, USA). All other chemicals were of analytical grade.

The Agilent 1100 series HPLC (Agilent technologies, Santa Clara, CA, USA) is used. The chromatography was performed on Shiseido (Tokyo, Japan) Capcell Pak MG $\mathrm{C}_{18}$ column $(50 \times 2.0 \mathrm{~mm}$ i.d., $3 \mu \mathrm{m}, 10 \mathrm{~nm})$. The mass spectrometry was performed on API 2000 mass spectrometer (AB Sciex, Toronto, Canada) equipped with Turboionspray ionization source. Data acquisition was performed with Analyst ${ }^{\mathrm{TM}} 1.5$ software (AB Sciex, Toronto, Canada).
(A)

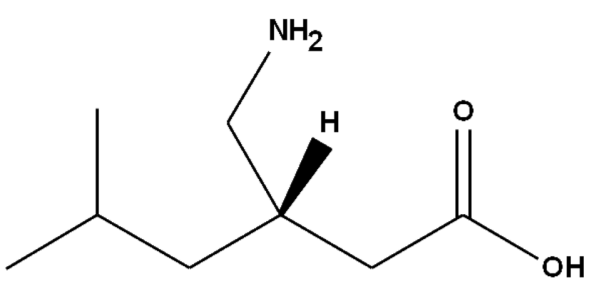

(B)

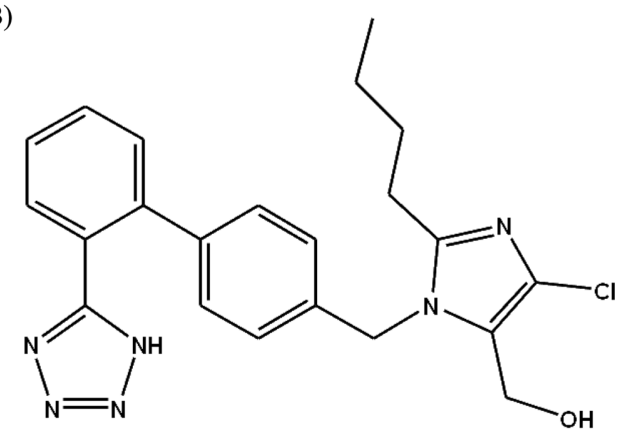

Figure 1. Chemical Structures for (A) pregabalin and (B) losartan (IS).

\section{Determination methods of pregabalin in human plasma Analytical conditions}

The mobile phase, which consisted of $10 \mathrm{mM}$ ammonium acetate buffer and acetonitrile $(15: 85, \mathrm{v} / \mathrm{v})$, was delivered at a flow rate of $0.2 \mathrm{~mL} / \mathrm{min}$ as an isocratic mode. The mobile phase was filtered through $0.22 \mu \mathrm{m}$ membrane and degassed prior to use. The stationary phase, Shiseido Capcell Pak MG $\mathrm{C}_{18}$ column, maintained at $45^{\circ} \mathrm{C}$.

The mass spectrometer was operated by electrospray ionization (ESI) at a positive ion mode. Typical source conditions were as follows: the ion spray voltage was set at $5.5 \mathrm{kV}$, and the source temperature was $500^{\circ} \mathrm{C}$. The curtain gas and collision-activated dissociation were 10 (arbitrary units), respectively. Ion source gas 1 was set at 45 (arbitrary units), ion source gas 2 was 65 (arbitrary units). Quantification was performed using the multiple reaction monitoring (MRM), with a dwell time of $200 \mathrm{~ms}$, transitions were $m / z \quad 160.1 \rightarrow 142.2$ for pregabalin, $m / z 423.2 \rightarrow 207.1$ for the IS, respectively. The optimized collision energy of $14 \mathrm{eV}$ was used for pregabalin and $32 \mathrm{eV}$ was used for the IS. The declustering potential (DP) was adjusted at 20 and 16 (arbitrary units) for pregabalin and IS, respectively. The ion mass spectra and the patterns of fragmentation of pregabalin and IS are presented in Figure 2.

\section{Preparation of the stock solution}

Standard stock solution of pregabalin $(1 \mathrm{mg} / \mathrm{mL})$ was prepared in methanol and IS $(1 \mathrm{mg} / \mathrm{mL})$ was prepared in acetonitrile. For calibration curve and quality control (QC) 
(A)

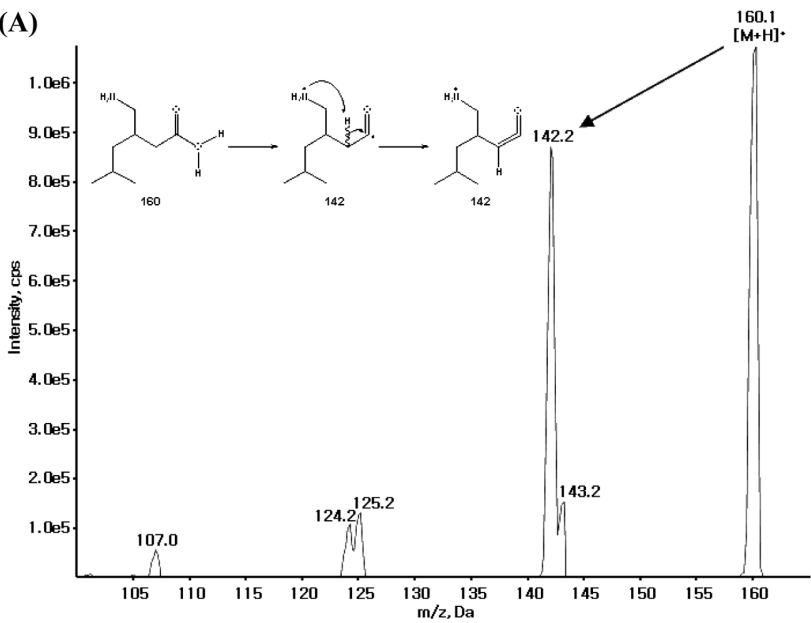

(B)

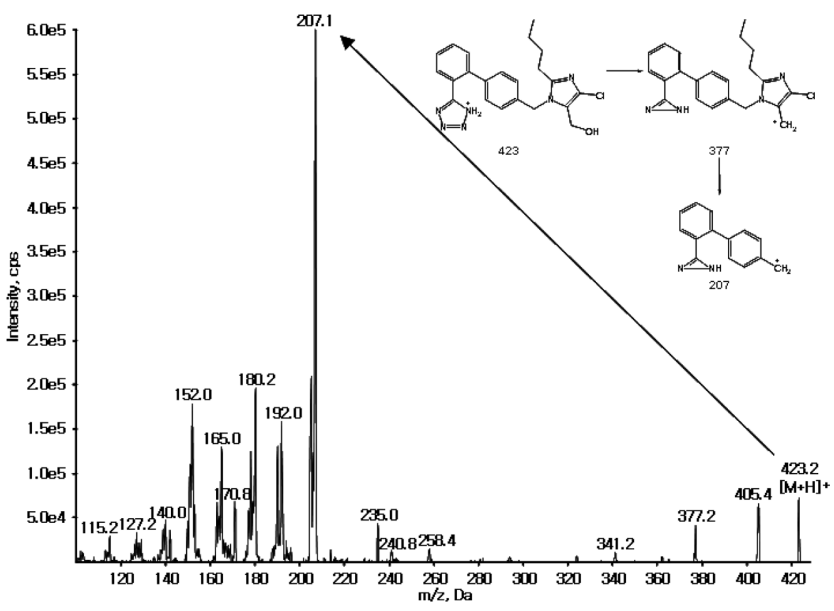

Figure 2. Product ion mass spectra and the patterns of fragmentation of (A) pregabalin and (B) losartan (IS).

sample, series of working standard solutions at concentrations of $1,2,3,5,10,20,50,80$ and $100 \mu \mathrm{g} / \mathrm{mL}$ were prepared by appropriate dilution of its stock with $50 \%$ methanol. Low-, medium-, high QC working standard solutions were 3, 20, $80 \mu \mathrm{g} / \mathrm{mL}$, respectively. An IS working solution $(10 \mu \mathrm{g} / \mathrm{mL})$ for routine use was freshly prepared by diluting stock solution with acetonitrile. A seven-point calibration curve was prepared by spiking the blank plasma with appropriate amounts of working standard solution to obtain final concentrations of 0.1 , $0.2,0.5,1,2,5$ and $10 \mu \mathrm{g} / \mathrm{mL}$ and quality control samples 0.3 , 2 and $8 \mu \mathrm{g} / \mathrm{mL}$ for analyte. All the solutions were stored at $20^{\circ} \mathrm{C}$ freezer when they were not in use.

\section{Sample preparation}

To $1.75 \mathrm{~mL}$ polypropylene tube, $80 \mu \mathrm{L}$ of human plasma sample was spiked with $20 \mu \mathrm{L}$ of IS working solution $(10 \mu \mathrm{g} /$ $\mathrm{mL}$ ) and $15 \mu \mathrm{L}$ of $10 \% o$-phosphoric acid. Then $1.2 \mathrm{~mL}$ of cold acetonitrile (stored in $-20^{\circ} \mathrm{C}$ ) was added and mixture was vortex-mixed for $15 \mathrm{~min}$. All the samples were centrifuged at $4^{\circ} \mathrm{C}, 14000 \mathrm{rpm}$ for $15 \mathrm{~min}$. The supernatant was filtered through a $0.20 \mu \mathrm{m}$ syringe filter. The resulting samples were loaded into the autosampler and $2 \mu \mathrm{L}$ aliquot of each sample was injected into the LC-MS/MS system for analysis.

\section{Method validation}

In accordance with Korea Food and Drug Administration (KFDA) guideline for the validation of bioanalytical method (KFDA, 2010b), the method was validated for specificity, sensitivity, linearity, precision, accuracy, recovery and stability.

\section{Specificity and sensitivity}

The specificity of the method was evaluated by analyzing drug-free blank plasma from six different subjects. There should not be any interference at the retention times of the analyte and IS. Specificity was assessed by comparing the chromatograms obtained from the blank plasma samples spiked with or without a pregabalin stock solution $(1 \mu \mathrm{g} / \mathrm{mL})$ and IS solution $(10 \mu \mathrm{g} / \mathrm{mL})$. The lower limit of quantification (LLOQ) was used to determine sensitivity and was defined as the concentration of pregabalin at which signal-to-noise ratio $(S / N$ ratio) was greater than 10 , with a precision less than $20 \%$ and accuracy between 80 and $120 \%$ of theoretical value. The LLOQ was set as the lowest concentration of calibration curve.

\section{Linearity}

The linearity of the calibration curves, ranging from 0.1 to $10 \mu \mathrm{g} / \mathrm{mL}$, was evaluated using a $1 / x^{2}$ weighed least-squares regression method of peak area ratios of pregabalin to IS versus actual concentrations. The acceptance criterion for a calibration curve was a correlation coefficient $(r)$ of 0.99 or better, and that each back-calculated standard concentration must be within $15 \%$ deviation from the theoretical value except for the LLOQ.

\section{Precision and accuracy}

To assess the intra- and inter-day precision and accuracy, assays were performed with five replicates on the same day and on four consecutive days. Pregabalin plasma samples at four concentration levels $(0.1,0.5,2$, and $10 \mu \mathrm{g} / \mathrm{mL})$ were analyzed to evaluate precision and accuracy of the method. Precision is expressed as coefficient of variance (C.V.) at each concentration. The accuracy of the assay was defined as a percentage of the experimental concentration over the theoretical concentration. The acceptance criterion recommended by KFDA for each back-calculated concentration was $15 \%$ deviation from the theoretical value except for LLOQ, which was set at $20 \%$. 


\section{Recovery and stability}

The extraction recovery of pregabalin (low-, medium- and high QC) and IS in human plasma were determined by comparing the peak area of precipitated analyte and IS with that of reference solutions at the same concentration level and reconstituted into blank plasma extracts. The number of replicates for each concentration was three.

The stability of the analyte (QC levels) in human plasma under different temperatures and time conditions was evaluated. For freeze-thaw stability, QC plasma samples were subjected to three cycles from $-70^{\circ} \mathrm{C}$ to room temperature. An autosampler stability was assessed by placing prepared QC samples in autosampler at $10^{\circ} \mathrm{C}$ for $27 \mathrm{hr}$, and long-term stability was evaluated by freezing QC plasma samples at $-70^{\circ} \mathrm{C}$ for ten days. Short-term stability was performed by placing QC plasma samples at different temperatures $\left(-70^{\circ} \mathrm{C}, 4^{\circ} \mathrm{C}\right.$ and room temperature) for $24 \mathrm{hr}$. Then the stability was assessed by comparing the area response of prepared stability samples with the area response of fresh QC plasma samples prepared without a storage period.

\section{Selection of volunteers}

The bioequivalence study population consisted of twenty eight healthy Korean volunteers with an average age of $24.53 \pm 4.47$ years and an average body weight of $73.97 \pm 5.82$ $\mathrm{kg}$. All of the participants were enrolled in the study after performing a medical history assessment, a physical examination and standard laboratory tests (blood analysis: WBC, RBC, hemoglobin, hematocrit, MCV, MCH, MCHC, MPV, platelet, differential counting of WBC, total protein, albumin, AST, ALT, ALP, total bilirubin, direct bilirubin, glucose, BUN, creatinine and total cholesterol, urine analysis: occult blood, bilirubin, urobilinogen, ketone, protein, nitrite, glucose, $\mathrm{pH}$, specific gravity, leucocytes, WBC and RBC). The subjects were excluded if they had possible sensitivity to pregabalin; had a history of gastrointestinal, hepatic, renal, cardiovascular, central nervous system, or endocrinal illness; or had ingested alcohol or any medications within 4 weeks before the study. Written informed consent was obtained from all subjects after the nature and details of the study had been explained, in accordance with the KFDA guideline for bioequivalence test (KFDA, 2010a).

\section{Blood sampling from volunteers}

The study protocol was approved by the Institutional Review Board of the Kyung Hee University. The study was performed in accordance with the revised Declaration of Helsinki and the Korean Good Clinical Practice (KGCP) guide- lines (KFDA, 2009).

The study had single-dose, randomized and $2 \times 2$ (two-treatment, two-period) crossover designs. The subjects were hospitalized (Kyung Hee University Medical Center, Seoul, Korea) at 6:30 p.m. on the eve of the study. And all of the subjects refrained from smoking, exercising, consuming xanthinecontaining foods, drinking alcoholic beverage overnight until 4 hr after each drug (test formulation: Daewoong pregabalin capsule, reference formulation: Lyrica ${ }^{\circledR}$ capsule) administration. The subjects received standardized meals at $4 \mathrm{hr}$ after drug administration. On the day of the study, at 7:00 a.m., a cannula was inserted into the subjects' median cubital vein and was flushed with heparinized normal saline to prevent clotting. At 8:00 a.m., each subject was given an oral dose (two pregabalin capsules; pregabalin $150 \mathrm{mg}$ ) with $240 \mathrm{~mL}$ of water. There was a one-week washout period between the different formulation doses. After $1.0 \mathrm{~mL}$ of blood was discarded, about $7.0 \mathrm{~mL}$ of blood samples were collected into heparinized tubes before $(0$ hr) and at 0.33, 0.67, 1, 1.5, 2, 2.5, 3, 4, 6, 8, 12 and $24 \mathrm{hr}$ after dosing. After sampling, the cannula was flushed with heparinized normal saline. Plasma was separated by centrifugation at 3,000 rpm for $10 \mathrm{~min}$ and kept frozen at $-70^{\circ} \mathrm{C}$ until analysis.

\section{Pharmacokinetics and statistical analysis}

After the single oral administration of $150 \mathrm{mg}$ of pregabalin capsules to subjects, pharmacokinetic parameters, including $\mathrm{AUC}_{0-\mathrm{t}}, \mathrm{C}_{\max }$ and $\mathrm{T}_{\max }$ were evaluated for the period of 0-24 hr by the measure of plasma concentration. The parameters were calculated by using BA calc $2007^{\circledR}$ program (Lee et al., 2007a) applying a non-compartmental method. $\mathrm{C}_{\max }$ and $\mathrm{T}_{\max }$ were recorded as actual measurement values and $\mathrm{AUC}_{0-\mathrm{t}}$ was calculated by linear-trapezoidal method from 0 to $24 \mathrm{hr}$. In addition, the ratios (test/reference) of $\mathrm{AUC}_{0-\mathrm{t}}$ and $\mathrm{C}_{\max }$ using logtransformed data, together with their means and $90 \%$ confidence intervals, were analyzed with the analysis of variance (ANOVA) that performed with the K-BE Test $2007^{\circledR}$ program (Lee et al., 2007b) at a significant level of 0.05. Bioequivalence of two formulations of pregabalin was assessed by $\mathrm{AUC}_{0-\mathrm{t}}$ and $\mathrm{C}_{\max }$ criteria of KFDA guideline for bioequivalence test (KFDA, 2010a).

\section{Results and Discussion}

\section{Validation results}

\section{Specificity and sensitivity}

Among the different possible detection techniques that can be coupled with LC, mass spectrometry has been unparalleled for performing bioanalytical determinations with maximum 
specificity and sensitivity. LC-MS/MS has high specificity because only ions derived from analytes of interest are monitored. Using drug-free plasma from six different subjects, blank plasma, plasma spiked with pregabalin stock solution $(1 \mu \mathrm{g} / \mathrm{mL})$, plasma spiked with IS solution $(10 \mu \mathrm{g} / \mathrm{mL})$ and pregabalin $0.1 \mu \mathrm{g} / \mathrm{mL}$ (LLOQ) plasma spiked with the IS were analyzed. The chromatograms for pregabalin and IS are presented in Figure 3. The peak retention time of pregabalin and IS were at 0.65 and $0.59 \mathrm{~min}$, respectively. No significant interference in the chromatograms was observed from endogenous substances in human plasma at the retention time of the analyte or IS. Also, there was no interference from the IS to the MRM channel of the analyte. The signal-to-noise ratio of LLOQ $(0.1 \mu \mathrm{g} / \mathrm{mL})$ was greater than 10 . The intra- and interday precisions for LLOQ were 6.02 and $11.04 \%$, respectively. The intra- and inter-day accuracies for LLOQ were 96.60 and $102.60 \%$, respectively.
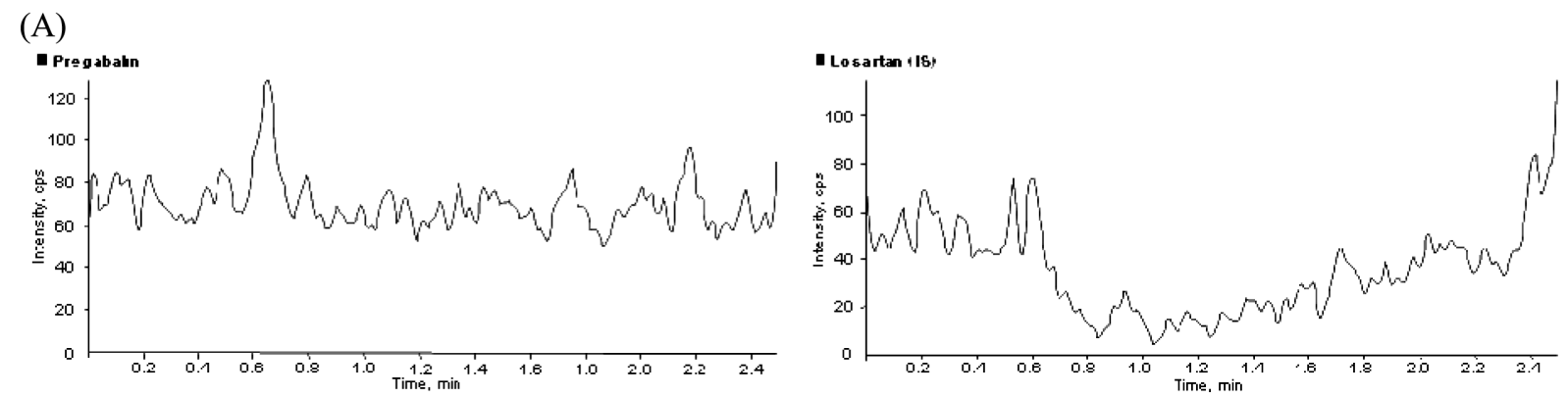

(B)
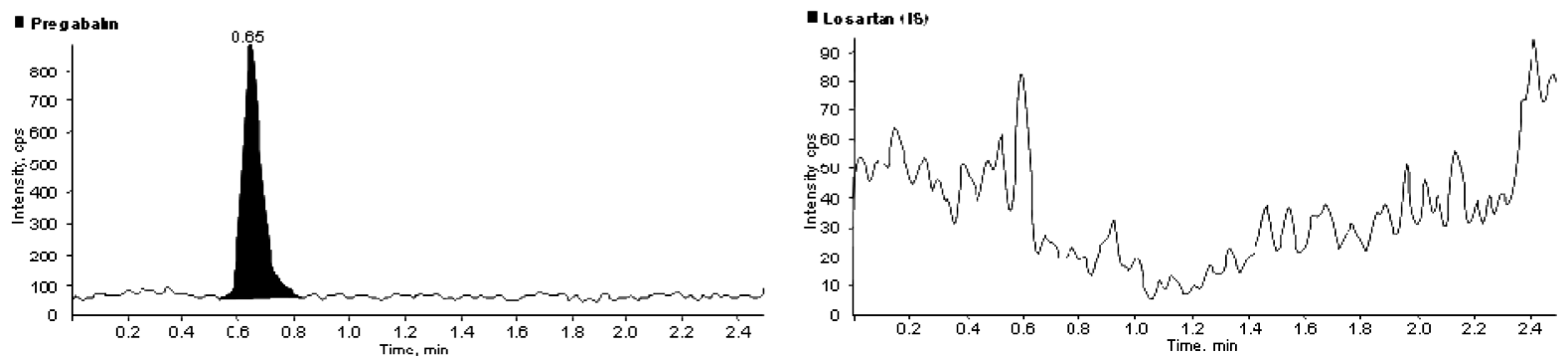

(C)
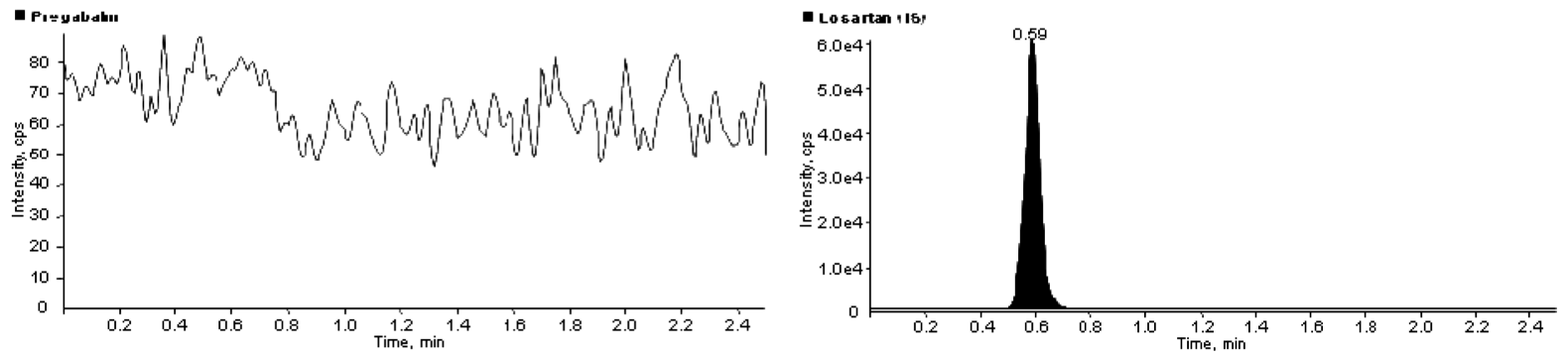

(D)
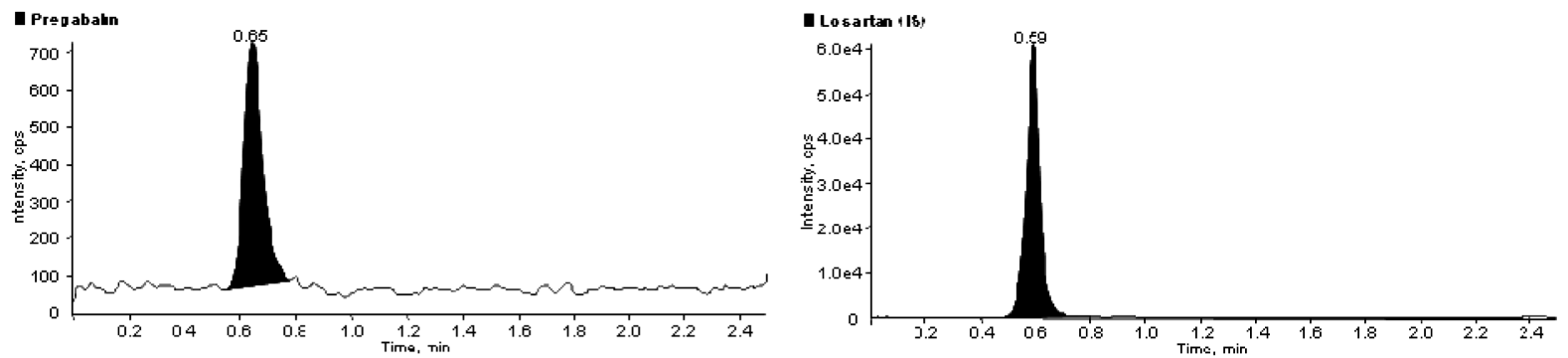

Figure 3. Multiple reaction monitoring chromatograms for pregabalin and IS of (A) blank human plasma, (B) plasma spiked with pregabalin $(1 \mu \mathrm{g} / \mathrm{mL})$, (C) plasma spiked with IS $(10 \mu \mathrm{g} / \mathrm{mL})$ and (D) $0.1 \mu \mathrm{g} / \mathrm{mL}$ (LLOQ) of pregabalin spiked with the IS. 


\section{Linearity}

To evaluate the linearity of the method, the seven-point calibration curve samples were analyzed on five independent runs. The equation of the calibration curve was obtained by a $1 / x^{2}$ weighed least-squares regression method of the peak area ratios of pregabalin to IS versus actual concentrations. The mean equation of all calibration curves $(n=5$, intra- and inter-day batches) obtained was $\mathrm{y}=(0.1352 \pm 0.008289)+(0.0008858$ $\pm 0.0008493)$, and the correlation coefficients $(r)$ were $\geq 0.9987$. And the each back-calculated standard concentration of all curves was within $15 \%$ deviation from the theoretical value. Thus, the calibration curves showed good linearity within the range of $0.1-10 \mu \mathrm{g} / \mathrm{mL}$. Results indicated the linearity and repeatability of the method.

\section{Precision and accuracy}

The intra- and inter-day precision and accuracy of the method used for pregabalin determination are presented in Table I. Intra-day C.V.s were $\leq 6.02 \%$, and inter-day C.V.s were $\leq 11.04 \%$. Intra- and inter-day accuracies ranged from 96.60 to $101.09 \%$ and from 98.10 to $102.60 \%$, respectively. All results were within the acceptance criteria of the KFDA guideline for the validation of bioanalytical method (KFDA, 2010b).

\section{Recovery and stability}

The mean extraction recoveries for pregabalin at QC concentrations after cold acetonitrile protein precipitation from human plasma were $81.58 \%, 81.66 \%$ and $80.29 \%$, respec- tively, and the mean extraction recovery of IS $(10 \mu \mathrm{g} / \mathrm{mL})$ was found to be $94.28 \%$.

The evaluated values of stability of the pregabalin in human plasma under different conditions are presented in Table II. Pregabalin stability after three freeze and thaw cycles was performed at QC levels and the percent bias was evaluated to be $6.30,1.49$ and $2.79 \%$, respectively. Autosampler stability was done for $27 \mathrm{hr}$ at the low-, medium-, high QC, respectively. The percent bias was evaluated to be $-1.57,-3.61$ and $-3.23 \%$, respectively. Long-term stability was done for ten days at $70^{\circ} \mathrm{C}$ and evaluated at a percent bias of $-5.51,-3.24$ and $-6.80 \%$ for QC levels, respectively. Short-term stability (24 hr) was performed at QC levels under different temperatures $\left(-70^{\circ} \mathrm{C}\right.$ and $\left.4^{\circ} \mathrm{C}\right)$. Pregabalin QC plasma samples that stored at $-70^{\circ} \mathrm{C}$ were evaluated at a percent bias of 5.51, 7.22 and 5.27\%, respectively. And short-term stability at $4^{\circ} \mathrm{C}$ was performed and the percent bias was evaluated to be 11.81, 15.94 and $11.26 \%$ for QC levels, respectively. No significant deterioration was observed under any of the examined conditions and then all the plasma samples of the bioequivalence study were stored at $-70^{\circ} \mathrm{C}$ and thawed at $4^{\circ} \mathrm{C}$.

\section{Pharmacokinetic analysis}

The devised method was successfully applied to analyze human plasma samples for a bioequivalence study of pregabalin $150 \mathrm{mg}$ capsules (test formulation: Daewoong pregabalin capsule, reference formulation: Lyrica ${ }^{\circledR}$ capsule) in twenty eight healthy Korean volunteers. Figure 4 presents the

Table I. Precision and accuracy of the method for determining pregabalin concentration in human plasma $(n=5)$

\begin{tabular}{|c|c|c|c|c|c|c|}
\hline \multirow[b]{2}{*}{$\begin{array}{l}\text { Theoretical } \\
\text { concentration } \\
(\mu \mathrm{g} / \mathrm{mL})\end{array}$} & \multicolumn{3}{|c|}{ Intra-day } & \multicolumn{3}{|c|}{ Inter-day } \\
\hline & $\begin{array}{c}\text { Experimental concentration } \\
(\text { mean } \pm \text { S.D. }) \\
(\mu \mathrm{g} / \mathrm{mL})\end{array}$ & $\begin{array}{l}\text { Precision } \\
(\%)\end{array}$ & $\begin{array}{c}\text { Accuracy } \\
(\%)\end{array}$ & $\begin{array}{c}\text { Experimental concentration } \\
(\text { mean } \pm \text { S.D. }) \\
(\mu \mathrm{g} / \mathrm{mL})\end{array}$ & $\begin{array}{c}\text { Precision } \\
(\%)\end{array}$ & $\begin{array}{c}\text { Accuracy } \\
(\%)\end{array}$ \\
\hline 0.1 & $0.097 \pm 0.006$ & 6.02 & 96.60 & $0.103 \pm 0.011$ & 11.04 & 102.60 \\
\hline 0.5 & $0.504 \pm 0.023$ & 4.49 & 100.84 & $0.502 \pm 0.012$ & 2.42 & 100.48 \\
\hline 2 & $1.983 \pm 0.042$ & 2.10 & 99.17 & $1.962 \pm 0.035$ & 1.79 & 98.10 \\
\hline 10 & $10.109 \pm 0.179$ & 1.77 & 101.09 & $9.837 \pm 0.335$ & 3.41 & 98.37 \\
\hline
\end{tabular}

Table II. Stability of pregabalin in human plasma under different temperatures and time conditions ( $n=3),($ C.V., \%)

\begin{tabular}{lccc}
\hline \hline \multicolumn{1}{c}{ Storage conditions } & Low-QC & Medium-QC & Medium-QC \\
$(0.3 \mu \mathrm{g} / \mathrm{mL})$ & $(2.0 \mu \mathrm{g} / \mathrm{mL})$ & $102.79 \%(2.28)$ \\
\hline 3 freeze/thaw cycles & $106.30 \%(2.22)$ & $101.49 \%(2.16)$ & $96.77 \%(0.74)$ \\
$27 \mathrm{hr}$ at autosampler $\left(10^{\circ} \mathrm{C}\right)$ & $98.43 \%(3.67)$ & $96.39 \%(0.67)$ & $93.20 \%(3.76)$ \\
10 days frozen $\left(-70^{\circ} \mathrm{C}\right)$ & $94.49 \%(5.00)$ & $96.76 \%(1.39)$ & $105.27 \%(0.51)$ \\
$24 \mathrm{hr}$ at $-70^{\circ} \mathrm{C}$ & $105.51 \%(1.29)$ & $107.22 \%(0.35)$ & $111.26 \%(4.37)$ \\
$24 \mathrm{hr}$ at $4^{\circ} \mathrm{C}$ & $111.81 \%(3.23)$ & $115.94 \%(3.62)$ & \\
\hline
\end{tabular}




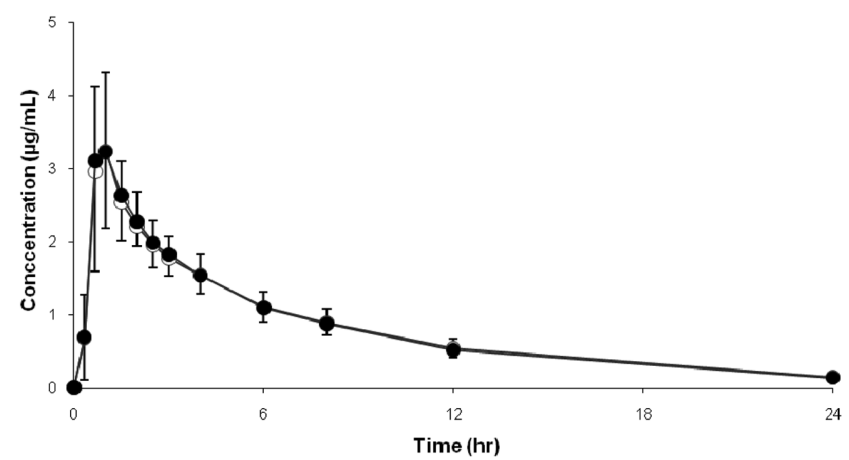

Figure 4. Mean plasma concentration-time profile of pregabalin from 28 healthy Korean volunteers after oral administration of a single $150 \mathrm{mg}$ of pregabalin capsule (, , reference formulation; $\bigcirc$, test formulation).

mean ( \pm S.D.) plasma concentration-time curve of pregabalin for test and reference formulations after the oral administration of a single dose of $150 \mathrm{mg}$ capsule, and the pharmacokinetic parameters derived from these profiles are presented in Table III. The pharmacokinetic parameters were calculated by lineartrapezoidal method in $0-24 \mathrm{hr}$. In this study, the mean ( \pm S.D.) $\mathrm{AUC}_{0 \text {-t }}$ was $18.70 \pm 4.25 \mu \mathrm{g} \cdot \mathrm{hr} / \mathrm{mL}$ for the reference formulation and $19.30 \pm 4.14 \mu \mathrm{g} \cdot \mathrm{hr} / \mathrm{mL}$ for the test formulation. The mean ( \pm S.D.) $\mathrm{C}_{\max }$ was $3.70 \pm 1.13 \mu \mathrm{g} / \mathrm{mL}$ for the reference formulation and the mean $\mathrm{T}_{\max }$ was $1.05 \pm 0.41 \mathrm{hr}$. The mean ( \pm S.D.) $\mathrm{C}_{\max }$ was $3.58 \pm 0.92 \mu \mathrm{g} / \mathrm{mL}$ for the test formulation and the mean $\mathrm{T}_{\max }$ was $1.19 \pm 0.72 \mathrm{hr}$.

\section{Bioequivalence study}

Bioequivalence analysis result is presented in Table IV. The $90 \%$ confidence intervals for the ratios of $\mathrm{AUC}_{0-\mathrm{t}}$ and $\mathrm{C}_{\max }$ were $\log 1.0048-\log 1.0692$ and $\log 0.9142-\log 1.0421$, respectively. The results met the bioequivalence criteria of KFDA $(\log 0.80-\log 1.25)$ (KFDA, 2010a), therefore, a test formulation (Daewoong pregabalin capsule; pregabalin $150 \mathrm{mg}$ ) is

Table III. Pharmacokinetic parameters obtained after oral administration of Lyrica ${ }^{\circledR}$ capsule and Daewoong pregabalin capsule at the dose of $150 \mathrm{mg}$ of pregabalin

\begin{tabular}{|c|c|c|c|c|c|c|c|c|c|c|}
\hline \multirow[b]{2}{*}{ Subjects } & \multicolumn{5}{|c|}{ Lyrica $^{\circledR}$ capsule } & \multicolumn{5}{|c|}{ Daewoong.pregabalin capsule } \\
\hline & $\begin{array}{c}\mathrm{AUC}_{0-\mathrm{t}} \\
(\mu \mathrm{g} \cdot \mathrm{hr} / \mathrm{mL})\end{array}$ & $\begin{array}{c}\log \\
\text { AUC }_{0-\mathrm{t}}\end{array}$ & $\begin{array}{c}\mathrm{C}_{\max } \\
(\mu \mathrm{g} / \mathrm{mL})\end{array}$ & $\begin{array}{l}\text { Log } \\
C_{\max }\end{array}$ & $\begin{array}{l}\mathrm{T}_{\max } \\
(\mathrm{hr})\end{array}$ & $\begin{array}{c}\mathrm{AUC}_{0-\mathrm{t}} \\
(\mu \mathrm{g} \cdot \mathrm{hr} / \mathrm{mL})\end{array}$ & $\begin{array}{c}\log \\
\text { AUC }_{0-\mathrm{t}}\end{array}$ & $\begin{array}{c}\mathrm{C}_{\max } \\
(\mu \mathrm{g} / \mathrm{mL})\end{array}$ & $\begin{array}{l}\log \\
C_{\max }\end{array}$ & $\begin{array}{l}\mathrm{T}_{\max } \\
(\mathrm{hr})\end{array}$ \\
\hline $\mathrm{A} 01$ & 17.34 & 1.24 & 3.00 & 0.48 & 1.50 & 19.14 & 1.28 & 3.02 & 0.48 & 1.00 \\
\hline A02 & 28.80 & 1.46 & 6.27 & 0.80 & 0.67 & 30.21 & 1.48 & 5.71 & 0.76 & 1.00 \\
\hline A03 & 22.19 & 1.35 & 5.52 & 0.74 & 0.67 & 20.64 & 1.31 & 4.24 & 0.63 & 0.67 \\
\hline A04 & 20.30 & 1.31 & 2.87 & 0.46 & 1.00 & 19.14 & 1.28 & 4.28 & 0.63 & 0.67 \\
\hline A05 & 24.61 & 1.39 & 4.08 & 0.61 & 1.50 & 26.78 & 1.43 & 4.78 & 0.68 & 1.00 \\
\hline A06 & 12.61 & 1.10 & 2.55 & 0.41 & 1.00 & 16.08 & 1.21 & 2.56 & 0.41 & 1.50 \\
\hline A07 & 20.59 & 1.31 & 4.87 & 0.69 & 1.00 & 18.64 & 1.27 & 4.16 & 0.62 & 1.00 \\
\hline A08 & 11.89 & 1.08 & 2.36 & 0.37 & 0.67 & 12.35 & 1.09 & 3.01 & 0.48 & 1.00 \\
\hline A09 & 11.75 & 1.07 & 2.90 & 0.46 & 1.00 & 14.48 & 1.16 & 2.29 & 0.36 & 1.00 \\
\hline A10 & 17.81 & 1.25 & 3.15 & 0.50 & 1.00 & 17.83 & 1.25 & 2.17 & 0.34 & 2.50 \\
\hline A11 & 19.15 & 1.28 & 2.64 & 0.42 & 1.50 & 20.31 & 1.31 & 2.99 & 0.48 & 1.00 \\
\hline A12 & 16.05 & 1.21 & 2.58 & 0.41 & 1.50 & 16.06 & 1.21 & 2.95 & 0.47 & 1.00 \\
\hline A13 & 12.91 & 1.11 & 3.38 & 0.53 & 1.00 & 16.27 & 1.21 & 3.88 & 0.59 & 0.67 \\
\hline A14 & 19.51 & 1.29 & 3.75 & 0.57 & 1.00 & 19.86 & 1.30 & 3.88 & 0.59 & 1.00 \\
\hline B01 & 13.64 & 1.13 & 4.56 & 0.66 & 0.67 & 12.75 & 1.11 & 4.10 & 0.61 & 1.00 \\
\hline B02 & 17.84 & 1.25 & 3.20 & 0.51 & 1.00 & 16.39 & 1.21 & 4.35 & 0.64 & 0.67 \\
\hline B03 & 22.75 & 1.36 & 3.75 & 0.57 & 1.00 & 23.56 & 1.37 & 3.31 & 0.52 & 1.50 \\
\hline B04 & 18.61 & 1.27 & 2.43 & 0.39 & 1.50 & 17.99 & 1.26 & 2.70 & 0.43 & 2.00 \\
\hline B05 & 17.08 & 1.23 & 3.70 & 0.57 & 1.00 & 16.43 & 1.22 & 3.12 & 0.49 & 0.67 \\
\hline B06 & 22.69 & 1.36 & 6.13 & 0.79 & 0.67 & 20.91 & 1.32 & 5.31 & 0.73 & 1.00 \\
\hline B07 & 20.53 & 1.31 & 3.06 & 0.49 & 1.50 & 21.96 & 1.34 & 3.44 & 0.54 & 0.67 \\
\hline B08 & 23.16 & 1.36 & 3.20 & 0.51 & 0.67 & 22.60 & 1.35 & 2.79 & 0.45 & 0.67 \\
\hline B09 & 22.49 & 1.35 & 5.51 & 0.74 & 0.67 & 22.79 & 1.36 & 4.16 & 0.62 & 0.67 \\
\hline B10 & 21.08 & 1.32 & 4.95 & 0.69 & 1.00 & 21.70 & 1.34 & 4.86 & 0.69 & 1.00 \\
\hline B11 & 19.48 & 1.29 & 3.98 & 0.60 & 0.67 & 20.80 & 1.32 & 3.17 & 0.50 & 1.50 \\
\hline B12 & 20.82 & 1.32 & 2.97 & 0.47 & 1.00 & 23.81 & 1.38 & 3.40 & 0.53 & 1.00 \\
\hline B13 & 13.19 & 1.12 & 2.92 & 0.47 & 2.50 & 13.05 & 1.12 & 3.31 & 0.52 & 2.00 \\
\hline B14 & 14.71 & 1.17 & 3.33 & 0.52 & 0.67 & 17.99 & 1.26 & 2.27 & 0.36 & 4.00 \\
\hline Mean & 18.70 & 1.26 & 3.70 & 0.55 & 1.05 & 19.30 & 1.28 & 3.58 & 0.54 & 1.19 \\
\hline S.D. & 4.25 & 0.10 & 1.13 & 0.12 & 0.41 & 4.14 & 0.09 & 0.92 & 0.11 & 0.72 \\
\hline
\end{tabular}


Table IV. Statistical results of bioequivalence evaluation between two pregabalin capsules

\begin{tabular}{ccc}
\hline \hline & \multicolumn{2}{c}{ Parameters } \\
\cline { 2 - 3 } & $\mathrm{AUC}_{0-\mathrm{t}}$ & $\mathrm{C}_{\max }$ \\
\hline Difference (\%) & 3.21 & -3.24 \\
Test/Reference estimate & 1.036 & 0.976 \\
90\% C.I. $(\delta)^{1)}$ & $\log 1.0048 \leq \delta \leq \log 1.0692$ & $\log 0.9142 \leq \delta \leq \log 1.0421$
\end{tabular}

${ }^{1)} \alpha=0.05$.

bioequivalent to a reference formulation (Lyrica ${ }^{\circledR}$ capsule; pregabalin $150 \mathrm{mg}$ ).

\section{Conclusion}

The described LC-MS/MS method for the determination of pregabalin in human plasma is successfully developed and validated. The method has proved to be rapid with each sample requiring a run time of $2.5 \mathrm{~min}$ only. And the sample preparation was simple one step protein precipitation. The method has shown acceptable specificity, sensitivity, linearity, precision and accuracy. In addition, this validated method was successfully applied to the bioequivalence study. And the statistical analysis results demonstrated that two different formulations of pregabalin were bioequivalent without significant differences in $\mathrm{AUC}_{0-\mathrm{t}}$ or $\mathrm{C}_{\max }$.

\section{References}

Dooley, D.J., Donovan, C.M., Meder, W.P., Whetzel, S.Z., 2002. Preferential action of gabapentin and pregabalin at $\mathrm{P} / \mathrm{Q}$-type voltage-sensitive calcium channels: inhibition of $\mathrm{K}^{+}$-evoked $[3 \mathrm{H}]$-norepinephrine release from rat neocortical slices. Synapse. 45, 171-190.

Dousa, M., Gibala, P., Lemr, K., 2010. Liquid chromatographic separation of pregabalin and its possible impurities with fluorescence detection after postcolumn derivatization with $o$ phtaldialdehyde. J. Pharm. Biomed. Anal. 53, 717-722.

Framton, J.E., Foster, R.H., 2005. Pregabalin: in the treatment of postherpetic neuralgia. Drugs. 65, 111-118.

Gee, N.S., Brown, J.P., Dissanayake, V.U.K., Offord, J., thurlow, R., Woodruff, G.N., 1996. The novel anticonvulsant drug, gabapentin (Neurontin), binds to the $\alpha_{2}-\delta$ subunit of a calcium channel. J. Biol. Chem. 271, 5768-5776.

Gray, P., 2007. Pregabalin in the management of central neuropathic pain. Expert Opin. Pharmacother. 8, 3035-3041.
Guay, D.R.P., 2005. Pregabalin in neuropathic pain: a more "pharmaceutically elegant" gabapentin? Am. J. Geriatr. Pharmacother. 3, 274-287.

Gujral, R.S., Haque, S.M. and Shanker, P., 2009. Development and validation of pregabalin in bulk, pharmaceutical formulations and in human urine samples by UV spectrophotometry. Int. J. Biomed. Sci. 5, 175-180.

Kasawar, G.B. and Farooqui, M.N., 2010. Development and validation of HPLC method for the determination of pregabalin in capsules. Indian. J. Pharm. Sci. 72, 517-519.

KFDA, 2009. Guideline for Korean Good Clinical Practice 2009211.

KFDA, 2010a. Guideline for Bioequivalence Test 2010-43.

KFDA, 2010b. Guideline for the Validation of Bioanalytical Method (2010. 06.).

Lee, Y.J., Chung, S.J., Shim, C.K., BA Calc $2007^{\circledR}$ for Windows ${ }^{\circledR}$, Version 1.0.0. (2007a)

Lee, Y.J., Chung, S.J., Shim, C.K., K-BE Test $2007^{\circledR}$ for Windows $^{\circledR}$, Version 1.1.0. (2007b)

Mandal, U., Sarkar, A.K., Gowda, K.V., Agarwal, S., Bose, A., Bhaumik, U., Ghosh, D., Pal, T.K., 2008. Determination of pregabalin in human plasma using LC-MS-MS. Chromatographia. 67, 237-243.

Maneuf, Y.P., Hughes, J., McKnight, A.T., 2001. Gabapentin inhibits the substance P-facilitated $\mathrm{K}^{+}$-evoked release of $\left[{ }^{3} \mathrm{H}\right]$ glutamate from rat caudal trigeminal nucleus slices. Pain. 93, 191-196.

Vaidya, V.V., Yetal, S.M., Roy, S.M.N., Gomes, N.A., Joshi, S.S., 2007. LC-MS-MS determination of pregabalin in human plasma. Chromatographia. 66, 925-928.

Vermeij, T.A.C., Edelbroek, P.M., 2004. Simultaneous high-performance liquid chromatographic analysis of pregabalin, gabapentin and vigabatrin in human serum by precolumn derivatization with $o$-phtaldialdehyde and fluorescence detection. J. Chromatogr. B. 810, 297-303.

Zareba, G., 2008. New treatment options in the management of fibromyalgia: role of pregabalin. Neuropsychiatr. Dis. Treat. 4, 1193-1201. 